\title{
EMPAGLIFLOZIN AMELIORATES TUNICA ADIPOSA EXPANSION AND VASCULAR STIFFENING OF THE DESCENDING AORTA IN FEMALE DB/DB MICE: AN ULTRASTRUCTURE STUDY
}

\author{
Melvin R. Hayden ${ }^{1-3^{*}}$
}

'Departments of Internal Medicine, ${ }^{2}$ Endocrinology Diabetes and Metabolism, ${ }^{3}$ Diabetes and Cardiovascular Disease Center, University of Missouri-Columbia School of Medicine, Columbia, Missouri, USA

\begin{abstract}
Background Obesity and aging are increasing globally and are known to be associated with adipose tissue and vascular stiffness, which are emerging as risk factors for the development of cardiometabolic and neurodegenerative diseases such as atherosclerosis, hypertension, type 2 diabetes mellitus (T2DM), metabolic syndrome, and Alzheimer's disease. Therefore, we wished to test the hypothesis that the descending thoracic aorta may demonstrate aberrant ultrastructural remodeling that is ameliorated by empagliflozin, a sodium/glucose cotransporter 2 (SGLT2) inhibitor.

Methods Ten-week-old female wild-type control (C57BLKS/J) and $d b / d b$ (BKS.Cg-Dock7m+/+Leprdb/J) mice were divided into three groups: lean untreated controls $(C K C, n=6)$, untreated $d b / d b$ controls $(D B C, n=6)$ and $D B C$ treated with empagliflozin (EMPA treated with $10 \mathrm{mg} / \mathrm{kg} /$ day for 10 weeks) (DBE, $\mathrm{n}=6)$.

Results This study focuses on ultrastructural remodeling of tunica adventitia and tunica adiposa (periadventitial adipose tissue) of the descending aorta in both DBC and DBE mice. In DBC mice (untreated $d b / d b$ controls), major observational remodeling included differentiation from thermogenic brown adipose tissue to white adipose tissue with hypertrophy of white adipocytes; ruptured plasma membranes and liberation of toxic lipids into the matrix, which incited inflammation with mast cells and macrophages. These changes were ameliorated in DBE mice (DBC treated with empagliflozin).

Conclusion Aberrant ultrastructural findings in DBC versus CKC and the amelioration with EMPA may provide better understanding how obesity and T2DM promote increased risk of vascular stiffness; a milieu for developing cardiovascular disease and target end-organ damage including nerve, retina, kidney and brain. Additionally, these findings may help us to better understand why obesity and T2DM result in the loss of homeostatic anticontractile function of tunica adiposa.
\end{abstract}

Adipobiology 2019; 10: 41-54

Keywords: aorta, adipose tissue, tunica adiposa (periadventitial adipose tissue), adventitia, $d b / d b$ mice, ultrastructure, vascular stiffness, mast cells

\footnotetext{
* Correspondence: Dr Melvin R. Hayden, Departments of Internal Medicine, Endocrinology Diabetes and Metabolism, Diabetes and Cardiovascular Disease Center, University of Missouri-Columbia School of Medicine, Columbia, Missouri, USA. E-mail: mrh29pete@gmail.com
} 


\section{Introduction}

The cardiometabolic diseases (CMD) such as atherosclerosis, hypertension, obesity, type 2 diabetes mellitus (T2DM) and metabolic syndrome (MetS) are increasing in prevalence globally and are expected to continue to increase during the coming decades (1). These conditions are associated with cardiometabolic disease and target end-organ diseases, which include diabetic neuropathy, retinopathy, cardiomyopathy, nephropathy, vasculopathy and diabetic cognopathy in addition to late onset Alzheimer's disease (LOAD) (2-9). The adventitia's outermost adipose tissue layer appears as a distinct entity and is commonly referred to as the perivascular adipose tissue (PVAT) or vascular periadventitial adipose tissue (PAAT), which was recently designated tunica adiposa (hereafter termed adiposa) comprised of adipocytes and stromal-vascular cells (10-12). Upon closer examination one can further discern two subdivisions of the adventitia from luminal side to the most abluminal side of the descending aorta: (i) tunica compacta (primarily comprised of type 1 collagen, and (ii) tunica lucida (primarily type III loose areolar collagen). Interestingly, the adiposa volume can exceed the volume of the entire descending aorta in the diabetic $d b$ / $d b$ aorta that in controls is comprised by a much thinner and almost exclusive thermogenic brown adipose tissue (BAT) of multilocular adipocytes.

Because of the marked aberrant remodeling in the adiposa, the latter became the major focus due to the marked differentiation of the multilocular thermogenic non-inflammatory BAT in control female (CKC) models to the unilocular pro-inflammatory white adipose tissue (WAT) in the female $d b / d b$ (DBC) adiposa. In the normal lean non-diabetic CKC thoracic descending aorta, the adiposa consists primarily of BAT; however, in the DBC the adiposa undergoes a near complete differentiation of BAT to WAT (Fig. 1).

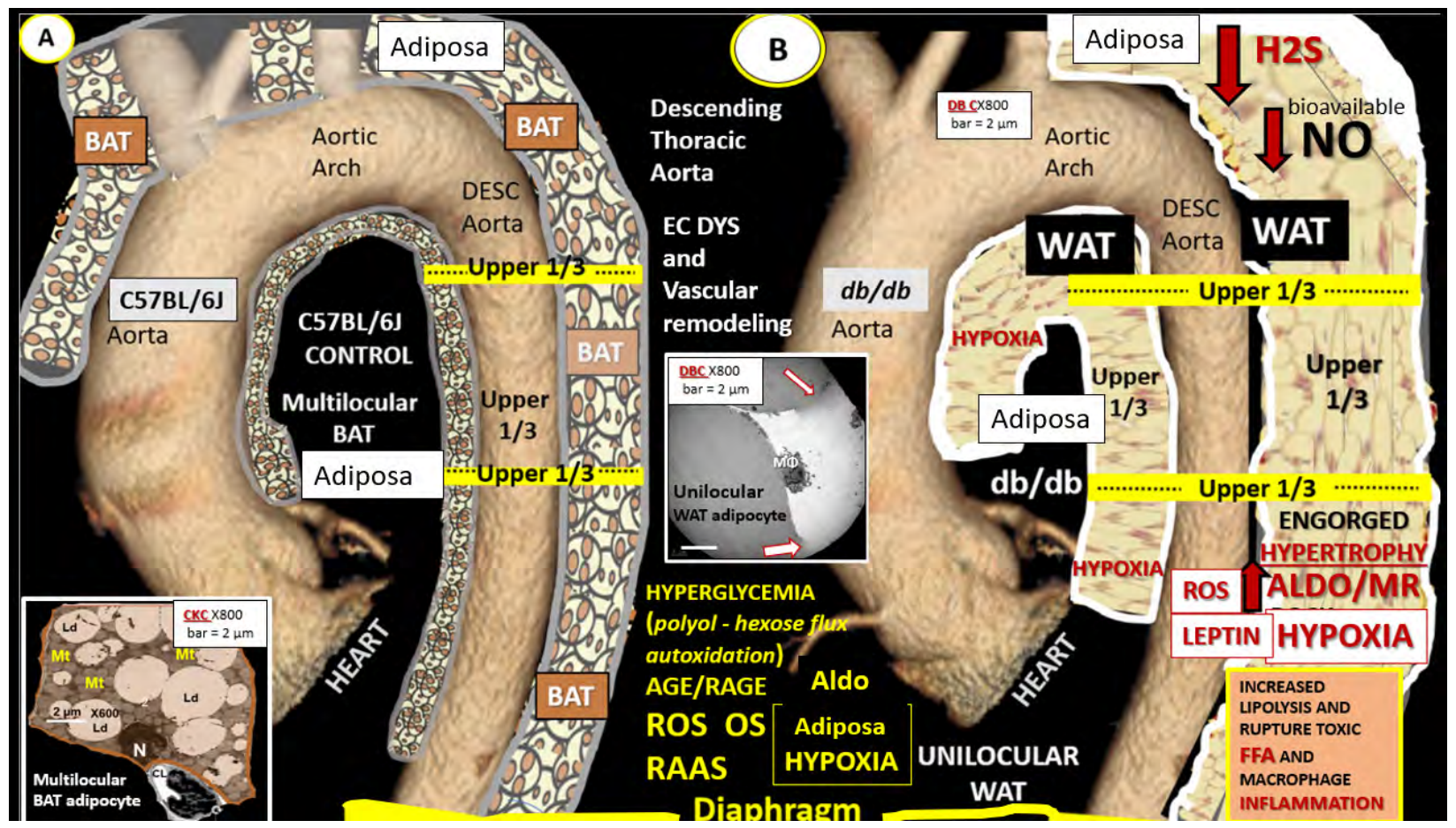

Figure 1. This illustration demonstrates the thermogenic multilocular brown adipose tissue (BAT) of the adiposa as in control CKC (panel A). In contrast, the DBC adiposa demonstrates marked and almost exclusive differentiation to unilocular white adipose tissue (WAT) (panel B). Notably, these two illustrations are not created to any scale. Interestingly, when the DBC is treated for 10-weeks with empagliflozin the adiposa is protected from this differentiation to WAT(not illustrated). Specimens for this ultrastructure study were obtained from the regions bounded by the two yellow bars labeled (Upper 1/3). Scale bars in transmission electron micrographs inserts $=2 \mu \mathrm{m}$; magnification $\times 800$ in panels $A$ and $B$. AGE, advanced glycation end products; ALDO, aldosterone; BAT, brown adipose tissue; DESC, descending aorta; EC DYS, endothelial dysfunction; FFA, free fatty acids; $\mathrm{H}_{2} \mathrm{~S}$, hydrogen sulfide; OS, oxidative stress; Ld, lipid droplets; MR, mineralocorticoid receptor; Mt, mitochondria; $M \Phi$, macrophage; NO, nitric oxide; RAGE, receptor for advanced glycation end products; RAAS, renin angiotensin-aldosterone system; ROS, reactive oxygen species; WAT, white adipose tissue. 
Since the initial recognition that adipose tissue, including vascular adiposa, is a highly active endocrine, paracrine and autocrine organ, there has been a substantial body of research devoted to this topic (10-12). In addition to the endocrine functions the adiposa structurally contains an stromal-vascular fraction, which is comprised of endothelial cells, pericytes and adipose precursor cells with resident mast cells as first responders to injury and the potential to assemble a large variety of circulatory inflammatory cells including mostly macrophages and fewer lymphocytes.

Conduit and resistant arterial stiffness in obesity and T2DM is an independent risk factor and strong predictor of CMD and LOAD along with the other multiple target end-organs (13). Additionally, aortic stiffness may be considered the nexus between cardiac, renal, brain and other end-organs associated with obesity and T2DM (13). Recently, Markus et al were able to demonstrate that elevations of glucose and insulin were positively associated with vascular stiffness (14). These multiple known abnormalities associated with obesity and T2DM along with the gross observations of excessive adipose tissue accumulation around the coronary, pericardial and thoracic aorta in diet-induced obesity female Western models and the $d b / d b$ models $(2,15,16)$ prompted this ultrastructural transmission electron microscopic (TEM) observational investigation of the diabetic $d b / d b$ thoracic aorta and treatment with empagliflozin, a SGLT2 inhibitor.

The goal of this study is to investigate the ultrastructural remodeling in the diabetic $d b / d b$ (DBC) conduit thoracic aorta as compared to the CKC and to observe if empagliflozin (EMPA) ameliorates aberrant remodeling since the effects EMPA treatment has been previously examined in the diabetic $d b / d b$ brain $(2,15,16)$.

\section{Materials and Methods}

\section{Animals and Treatments}

Three cohorts of mice were used: lean non-diabetic controls (CKC, $n=5$ ), obese, insulin-resistant and diabetic $d b$ / $d b(\mathrm{DBC}, n=6)$, and $d b / d b$ mice treated with the SGLT2 inhibitor, EMPA, to deliver $10 \mathrm{mg} \mathrm{kg}^{-1} \mathrm{day}^{-1}$ and fed for five-weeks, initiated at 10 weeks of age (DBE, $n=6)$. All mice were sacrificed for study at 20 weeks of age. All animal studies were approved by the Institutional Animal Care and Use Committees at the Harry S Truman Memorial Veterans' Hospital and University of Missouri, Columbia, MO, USA (No.190), and conformed to the Guide for the Care and Use of Laboratory Animals published by the National Institutes of Health (NIH).

Eight-week-old female $d b / d b$ (BKS.Cg-Dock $7^{\mathrm{m}}+/+L e p r^{d b} / \mathrm{J}$;
DBC) and wild-type control (C57BLKS/J; CKC) mice were purchased from the Jackson Laboratory (Ann Harbor, MI, USA) and were housed under standard laboratory conditions where room temperature was $21-22^{\circ} \mathrm{C}$, and light and dark cycles were $12 \mathrm{~h}$ each. Three cohorts of mice were used: lean non-diabetic controls (CKC, $n=6$ ), obese, insulin-resistant and diabetic $d b$ / $d b$ (DBC, $n=6)$, and $d b / d b$ mice treated with the SGLT2 inhibitor, EMPA, to deliver $10 \mathrm{mg} \mathrm{kg}^{-1} \mathrm{day}^{-1}$ and fed for 10 -weeks, initiated at 10 -weeks of age (DBE, $n=6)$. All mice were sacrificed for study at 20 weeks of age. We chose female mice because the cardiovascular phenotype has been previously characterized (15).

\section{Tissue Collection and Preparation for Transmission Electron Microscopy (TEM)}

Upon sacrifice (at 20-weeks of age) approximately 1-mm aortic ring slices from the upper $1 / 3$ of the descending thoracic aorta of CKC, DBC and DBE were immediately immersion-fixed and placed in a standard TEM fixative of $2 \%$ paraformaldehyde and $2 \%$ glutaraldehyde in $100 \mathrm{mM}$ of sodium cacodylate buffer $(\mathrm{pH}=7.35)$ for immersion fixation. These specimens were then rinsed with $100 \mathrm{nM}$ sodium cacodylate buffer ( $\mathrm{pH} 7.35$ ) containing $130 \mathrm{mM}$ sucrose. Secondary fixation was performed using $1 \%$ osmium tetroxide (Ted Pella, Inc., Redding, CA, USA) in cacodylate buffer using a Pelco Biowave (Ted Pella) operated at $100 \mathrm{~W}$ for $1 \mathrm{~min}$. Specimens were next incubated at $4^{\circ} \mathrm{C}$ for $1 \mathrm{~h}$, then rinsed with cacodylate buffer, and further rinsed with distilled water. En bloc staining was performed using 1\% aqueous uranyl acetate and incubated at $4{ }^{\circ} \mathrm{C}$ overnight, then rinsed with distilled water. Using the Pelco Biowave, a graded dehydration series (e.g., $100 \mathrm{~W}$ for $40 \mathrm{~s}$ ) was performed using ethanol, transitioned into acetone, and dehydrated tissues were then infiltrated with Epon resin (250 W for $3 \mathrm{~min}$ ) and polymerized at $60{ }^{\circ} \mathrm{C}$ overnight. Ultrathin sections were cut to a thickness of $85 \mathrm{~nm}$ using an ultramicrotome (Ultracut UCT, Leica Microsystems, Wetzlar, Germany) and stained using Sato's triple lead solution stain and 5\% aqueous uranyl acetate. Multiple images were acquired for study group at various magnifications with a JOEL 1400-EX TEM JEOL (JEOL, Peabody, MA, USA) at $80 \mathrm{kV}$ on a Gatan Ultrascan 1000 CCD (Gatan, Inc., Pleasanton, CA, USA). ds. Incidentally, these aortas were from the same model that we studied the diabetic $d b / d b$ DBC brain (16). Of note, all of these specimens were preferably immersion fixed because we were also studying brains in these animals via immersion fixation in order to compare to human brain biopsies and/or rapid autopsy specimens to increase clinical translation of preclinical rodent models. 


\section{Results}

Light microscopy and low TEM magnification of the upper $1 / 3$ descending thoracic aorta

We routinely examine toluidine blue stained slides prior to TEM preparations in order to localize our tissues and in this study, we were able to note a marked difference in remodeling between the control CKC and $d b / d b$ diabetic DBC. Observations indicated an approximately three-fold increase in volume of the adiposa of DBC as compared to the CKC thinner BAT adiposa (Fig. 2). Additionally, TEM sections were examined at low magnifications and assembled into a collage of images to visualize the ultrastructural morphology of the control descending thoracic aorta (Fig. 3).

\section{Descending aorta tunica intima}

Previously, the DBC showed increased glucose levels (HbA1c), blood pressure, aortic and endothelial cell (EC) stiffness, and impaired endothelium-dependent vasorelaxation (2). This study demonstrated impaired activation of endothelial nitric oxide synthase enzyme (eNOS) and revealed that the SGLT2 inhibitor empagliflozin promoted glycosuria and blunted vascular and renal abnormalities without raising or lowering blood pressure. TEM images revealed significant shortening and contraction, separation, lifting and apoptosis in the DBC as compared to the elongated ECs in CKC. Also, this study was able to demonstrate that EMPA treatment in the DBE for last 10-weeks prior to sacrifice was able to significantly improve the length of ECs in association with a significant decrease in aortic vascular stiffening (2).

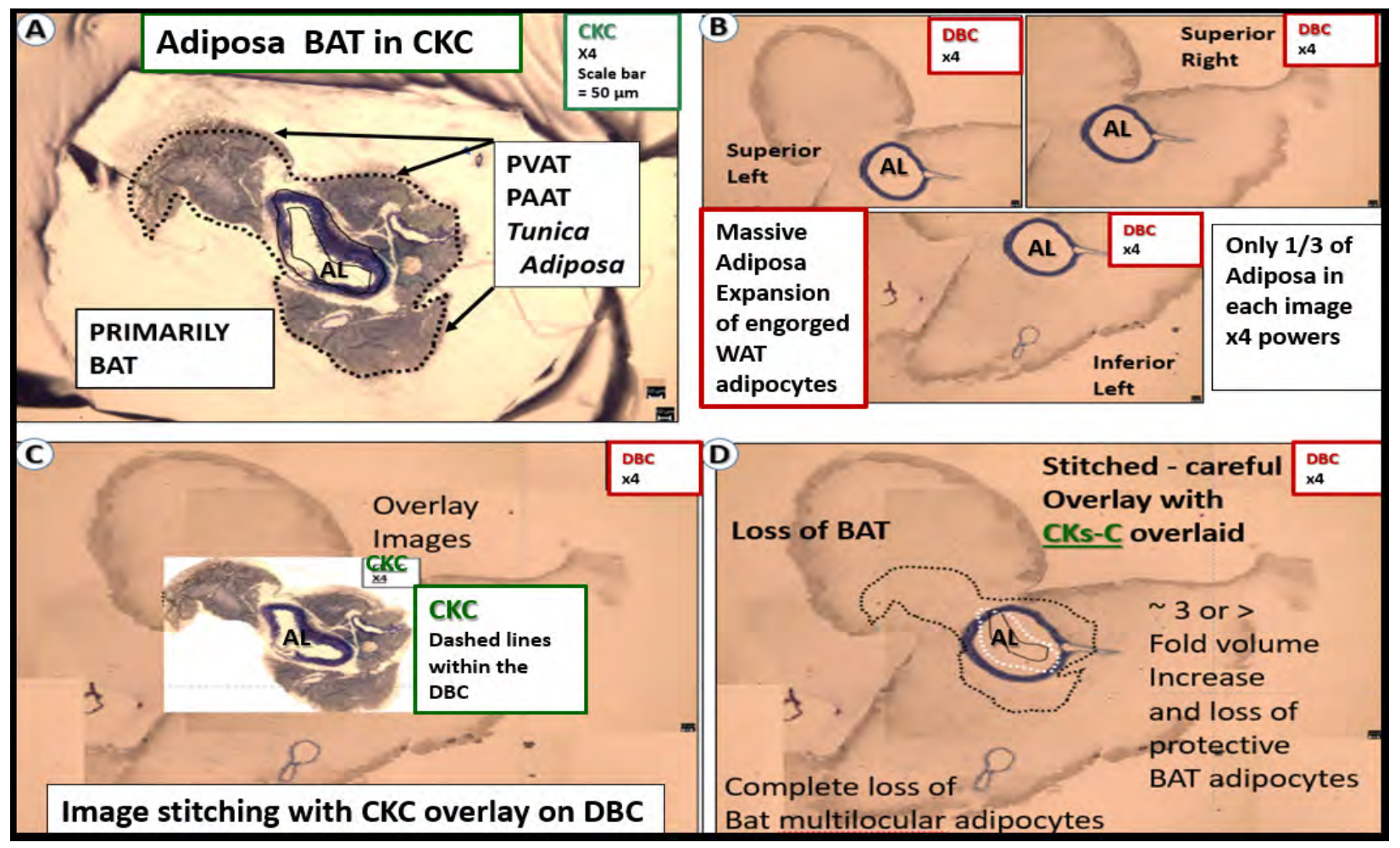

Figure 2. Brown adipose tissue differentiates to white adipose tissue in the tunica adiposa of diabetic $d b / d b$ at 20-weeks. Panel A illustrates the control CKC aorta and note the dark staining of the BAT (dashed lines) adiposa. Centrally there is some WAT unilocular adipocytes; however, they are hard to discern at the periphery of the BAT. Note how easy it is to discern the special phenotype of the tunica adiposa surrounding the aorta. Panel B depicts the dramatic differentiation of BAT in panel A to WAT (BAT "whitening") in the diabetic $\mathrm{db} / \mathrm{db}(\mathrm{DBC})$ in panel B and importantly note the $\sim 3$-fold increase in volume of the adiposa. Panel C illustrates the difference in volume between the CKC and the DBC when the CKC control is overlaid and stitched in the DBC model. Panel D reveals the marked expansion of the DBC's volume in addition to its complete differentiation from BAT to WAT. Unfortunately, we do not have the comparable images of the models treated with empagliflozin (DBE). Toluidine blue staining Panels A-D. Magnification objective x4; scale bar very small =10 $\mu \mathrm{m}$. $A L=$ aortic lumen; BAT = brown adipose tissue; PAAT, periadventitial adipose tissue; PVAT. perivascular adipose tissue; WAT, white adipose tissue. 


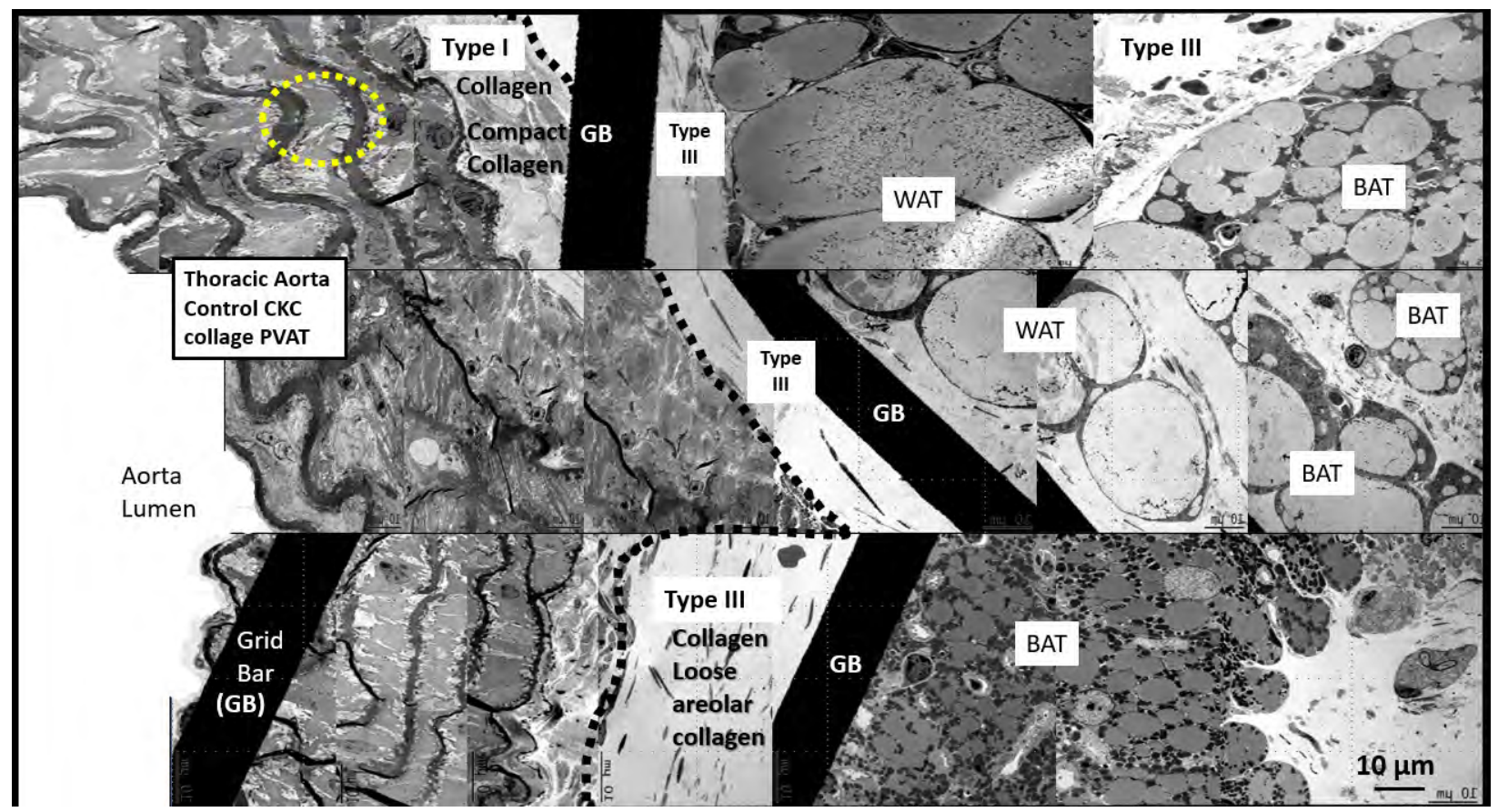

Figure 3. Transmission electron micrograph collage of descending thoracic aorta. This collage depicts each tunica coating of the aorta. As one passes from the lumen tunica intima to the multiple layers of the vascular smooth muscle cells (VSMCs) of the tunica media (note the elastin lamellar unit is outlined by yellow dashed lines) to the most abluminal adventitia composed of the compact dense collagen type I to the loose areolar type III collagen that gradually gives way to the discernible phenotypic abluminal tunica adiposa. The adiposa is sparsely decorated by white adipose tissue (WAT) and unilocular adipocytes at its periphery and is composed almost entirely of multilocular brown adipose tissue (BAT) adipocytes. Note that both BAT and WAT are illustrated with BAT adipocytes have multilocular lipid droplets and increased numbers of electron dense mitochondria as compared to the unilocular white adipocytes. Each section of at least 12 individual images compose this mosaic collage and each has a scale bar $=10 \mu \mathrm{m}$. PVAT, perivascular adipose tissue or tunica adiposa.

Descending aorta tunica intima elastolysis and elastin-derived products

Elastin is a long-lived polymer with monomeric precursor tropoelastin and allows conduit and some resistant arteries with an internal elastic lamina and elastic lamella to serve as an elastin recoil pump associated with elastic recoil and maintenance of pulse pressure, pulse wave velocity in vascular hemodynamics. When elastin is damaged due to aging and accumulating diabetic metabolic injuries there is a loss of this recoil function and these vessels become stiffened with an associated increase in pulse wave velocity and pulse pressure which is associated with aortic and vascular stiffening.

Elastolysis implies degradation by elastases, including those from mast cells, macrophages and lymphocytes, and are strongly associated with inflammaging (17). Elastolysis was observed in the DBC models and associated with markedly electron dense protein staining that could be the result of accumulated frag- mented proteins due to elastolysis, which could represent elastin-derived peptide(s) (EDP). These electron densities were associated with separation of the endothelial cells (endothelium) from the internal elastin lamina that were not noted in the CKC and DBE (Fig. 4, 5).

Importantly, activation of ROS via hyperglycemia (polyol hexosamine flux - glucose autoxidation), advanced glycation products (AGE) and receptor to AGE (RAGE) - AGE/RAGE interactions and subsequent matrix metalloproteinase (MMP) and cathepsin cysteine proteases, such as cathepsin S upregulation and activation may also be responsible for the observed elastolysis and EDPs.

Elastin-derived peptide(s) created as a result of elastolysis and elastin degradation, which are associated with inflammation may accelerate aging and promote increased vascular stiffening in diet induced obesity Western obese mice and $d b / d b$ diabetic models and in Western models transforming growth factor beta 


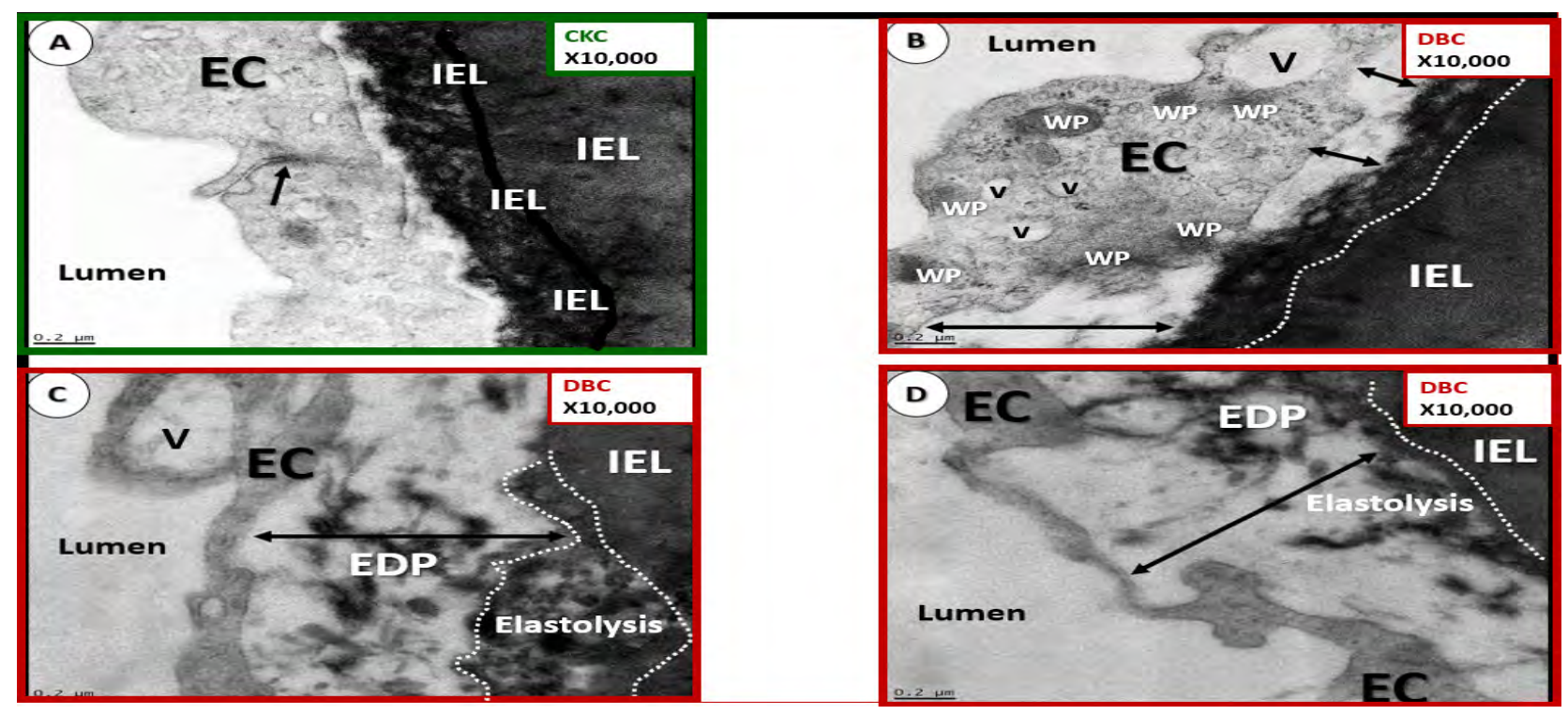

Figure 4. Elastolysis and elastin-derived products (EDP) in diabetic DBC.

Panel A illustrates the normal appearance of the endothelial cell in relation to the internal elastic lamina (IEL) in control CKC and note the tight adherence of the endothelial cells (EC) to the IEL. Also note the prominent adherens junction of the vascular endothelial cadherin (VE-Cadherin) (arrow). Panel B-D depict marked remodeling changes in the obese diabetic DBC models as compared to control image in Panel A. These phenotypic changes may depict activation and dysfunction of ECs. Panel B depicts the vacuole $(\mathrm{V})$ and vesicles $(\mathrm{v})$ in the $\mathrm{EC}$ along with an increase in weibel palade bodies (WP) with separation of the $\mathrm{EC}$ from the IEL (double arrows). Panel C depicts EC thinning and even further separation from the IEL (double arrows) and importantly note the marked electron dense bodies within the intimal space that may represent IEL fragments of EDPs in this area of elastolysis, which are also present in panel D. Magnification $\times 10000$; scale bar $=0.2 \mu \mathrm{m}$.
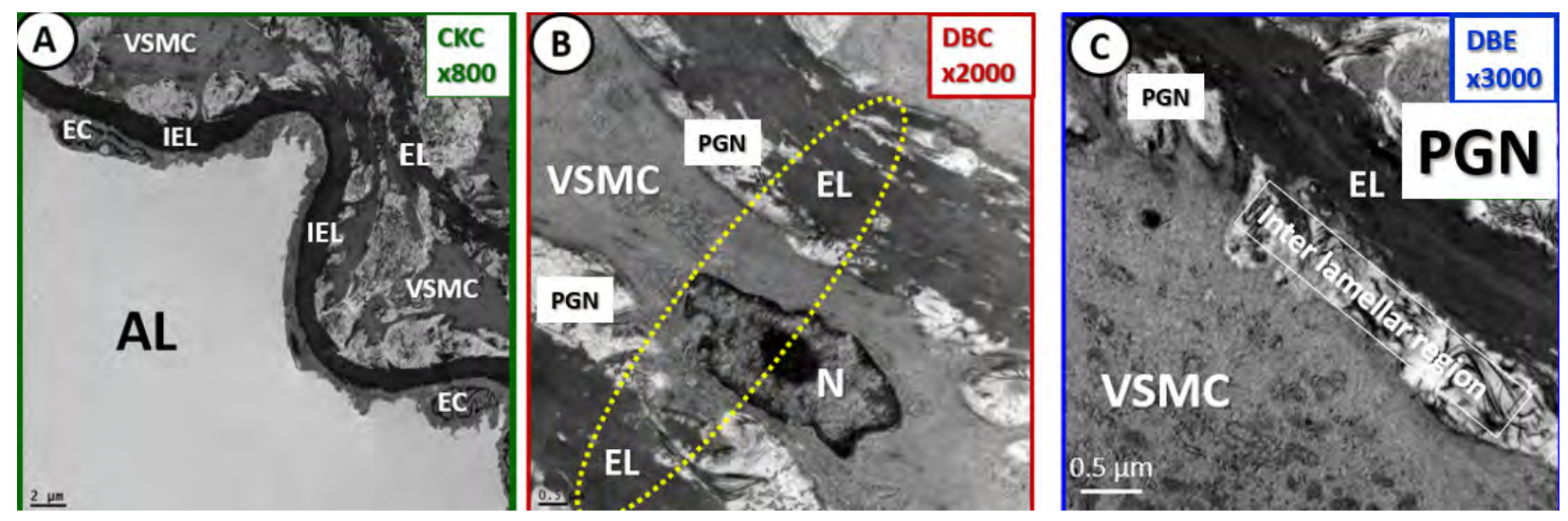

Figure 5. Addition layering of elastin in DBC ameliorated with empagliflozin. Panel A illustrates the tightly adherent endothelial cell (EC) to the internal elastic lamina (IEL) and low magnification was depicted purposefully to show more of the endothelium. Panel B depicts not only degradation of elastin lamella (EL) but also the 'addition layering phenomenon' of elastin as well as the functional lamellar unit in the DBC (outlined in yellow dashed oval). This representative image in panel B was a constant finding in the aortas in the DBC models. Panel $\mathbf{C}$ reveals the amelioration of elastin lamella degradation as well as the lack of addition layering of elastin. Varying magnifications in upper corner and scale bars depicted lower corner of each image. $\mathrm{AL}$, aortic lumen; PGN, proteoglycan; VSMC, vascular smooth muscle cell. 
and neuramidase were increased (18). Importantly, elastolysis and formation of EDPs (electron dense elastin fragments) were not observed in the CKC or the empagliflozin treated DBE.

\section{Descending thoracic 'additional layering' of elastin lamella in tunica media}

There were no observations of any major remodeling changes such as matrix proteoglycan expansion as in the lean hypertensive Ren 2 model in the $\mathrm{DBC}$ as compared to the CKC control in regard to vascular smooth muscle cells within the media (19). However, observations demonstrated marked remodeling changes not only to the internal elastic lamina but also to the elastin lamella and the lamellar units within the tunica media of the DBC. Each elastin lamella layer demonstrated degradation and an 'additional layering phenomenon' of elastin to the elastin lamellar units of the media (Fig. 5). While there are definite observational changes of degradation/elastolysis to the internal elastin lamina and the lamellar units in DBC there is also known to be an associated increase in lysyl oxidase, which may be responsible for the crosslinking of elastin in the $d b / d b$ diabetic with hyperglycemia and this may be related to the additional layering phenomenon' of the elastin lamella of the lamellar units observed as occurred in the response to injury mechanisms in DBC (20).

\section{Tunica adiposa remodeling in female diabetic $d b / d b$ DBC}

The descending thoracic aorta adiposa in CKC and healthy humans is normally constituted by thermogenic, anti-inflammatory multilocular BAT adipocytes and our specimens were specifically obtained from the upper $1 / 3$ of the descending aorta, This finding in the thoracic aorta is in contrast to the abdominal aorta, which is known to be comprised entirely of proinflammatory unilocular white adipose tissue in health and controls (21). However, during the development of obesity, insulin resistance, leptin resistance and T2DM the BAT in CKC descending aorta differentiates to large unilocular proinflammatory WAT adipocyte phenotypes in DBC. Further, these adipocytes undergo marked engorgement due to storage of excessive nutrient intake due to hyperphagia associated with lack of cellular signaling of the satiety hormone leptin. This is thought to result in the development of huge hypertrophic adipocytes with expansion of lipid droplets by gorging until the outer plasma membrane thins with loss of organelles (Fig. 6). This thinning and engorgement of adipocytes continue until their plasma membrane rupture and allows for the release of toxic lipids (free fatty acids, sphingolipids, diacylglycerol (DAG) and ceramides) into the interstitial matrix. The presence of these toxic lipids in the interstitial matrix incites acute and eventually chronic inflammation with macrophage accumulation. This chronic infiltration of multiple inflammatory cells are largely due to the increase in monocyte chemoattractant protein-1 (MCP-1) as in diet-induced obesity Western preclinical models (22) plus other factors that may be responsible for the further aberrant remodeling changes within the adiposa of descending thoracic aortas (Fig. 6).

There is considerable plasticity in mature adipocytes in order to maintain homeostasis in response to their environment (22). The previous aberrant remodeling images (Figs. 1, 2, 6) depicting differentiation of BAT to WAT adipocyte phenotypes is visible evidence of this plasticity. This plasticity is illustrated and discussed in greater depth by Grigoras et al (23). The following illustration attempts to assist in the understanding of the related known vascular aortic stiffening that is noted in DBC, which is founded on this plasticity (Fig. 7).

There is extreme thinning of the plasma membrane in the DBC ( 0.5 to $0.75 \mathrm{~nm})$ as compared to the 0.5 to $1 \mu \mathrm{m}$ in CKC and DBE. This extreme thinning and loss of organelles including the mitochondria may indeed be the structural basis for the DBC adipocytes being prone to rupture (similar to blowing up a balloon until it bursts).

Thinning of the WAT adipocyte plasma membrane and rupture appears to be associated with regions of extreme electron densities within the plasma membrane and associated with the loss of cytoplasmic organelles in DBC. These similar findings have been noted by Giordano et al (24) in $\mathrm{db} / \mathrm{db}$ models of obesity/T2DM. They stained WAT adipocytes in culture and in vivo with calcium von Kossa stain to demonstrate that hypertrophic WATs stained positive for calcium and to further demonstrate that these adipocytes depict ultrastructural features of stressed cells and may die of pyropoptosis (an inflammatory mode of cell death). They also were able to demonstrate that these WAT adipocytes were associated with crown like structures (CLS) of macrophages similar to our studies. Additionally, this study also demonstrated the presence of other inflammatory cells including mast cells and occasional lymphocytes.

\section{Proinflammatory and profibrotic tunica adiposa in diabetic $d b / d b$ DBC aorta}

There were occasional resident first responder mast cells in the adiposa of control CKC models, but one had to hunt extensively to find macrophages and even more so to observe lymphocytes; however, in the diet-induced obesity models such as the Western model fed a high sucrose/fructose and high fat diet one could readily find a host of inflammatory cells due to obesity, insulin resistance and impaired glucose tolerance. In both the Western and the DBC models there is an interaction of immune cells and adipocytes (11) 


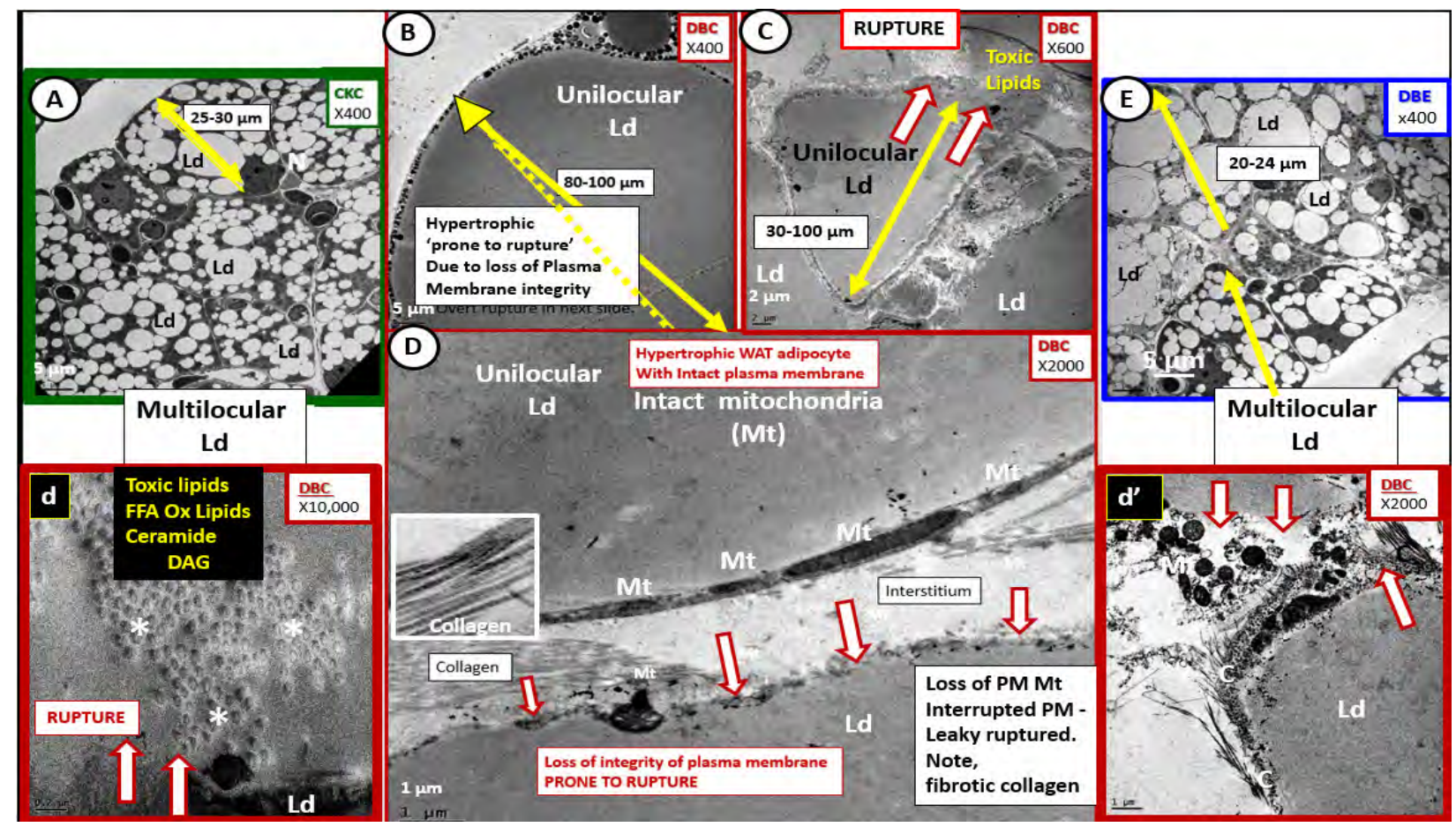

Figure 6. Multilocular brown adipose tissue (BAT) in control CKC differentiates to unilocular white adipose tissue (WAT) in diabetic DBC. Panel A illustrates the normal tunica adiposa in the control CKC and note the multilocular BAT adipocytes with diameter between 25-30 micrometers. Panels B and C depict the complete differentiation from multilocular BAT adipocytes to unilocular WAT adipocytes and note the hypertrophy between 80-100 micrometer as compared to the CKC in panel A (yellow double arrows). Panel $\mathbf{C}$ depicts a rupture of the unilocular WAT adipocyte in diabetic $\mathrm{db} / \mathrm{db}$ DBC models (red open arrows). Panel D illustrates that not all hypertrophic WAT adipocytes are in the process of rupture and note the two adjacent adipocytes with the upper one displaying an intact plasma membrane (PM) with intact electron dense mitochondria (Mt) and the lower adipocyte depicting a loss of integrity with multiple interruptions or loss of the plasma membrane (PM) (open red arrows). Panel E demonstrates that 10 weeks of empagliflozin treatment protects the BAT adipocyte from undergoing the differentiation as illustrated in the DBC models (Panels B-D). Importantly, note the accumulation of toxic lipid extrusions (asterisks) in panel $d$. Further, note the accumulation of collagen within the interstitium between these two adipocytes ( $D$ and d). Also, note the marked electron densities in the plasma membranes which might represent calcium depositions as discussed later. DAG, diacylglycerol; FFA, free fatty acids; Ld = lipid droplet(s); Ox, oxidized.

In the DBC, the WAT unilocular adipocyte models revealed an even greater inflammatory response with macrophage crown like structures (CLS) in a response to injury of the plasma membrane with plasma membrane thinning, loss of organelles/mitochondria and rupture of engorged WAT adipocytes (Figs. 8-10). When the thinned adipocyte ruptures it allows toxic FFA, oxidized lipids, ceramides and diacylglycerol (DAG) to leak from the adipocytes and be expelled into the interstitial matrix, which incite the first responder mast cells and a monocyte chemotactic protein - 1 (MCP-1) chemokine response and activate the ingress of inflammatory macrophages, which dependently or independently may also incite lymphocyte cell ingress (2528). Importantly, the chronic inflammation with macrophages are capable of forming what are commonly termed crown-like structures (CLS) (Fig. 8). Importantly, these findings were not present in the empagliflozin treated DBE, since they are protected from the differentiation of multilocular, thermogenic and anti-inflammatory BAT adipocytes to unilocular proinflammatory WAT adipocytes.

\section{Discussion}

While the focus of this observational study has been primarily adiposa and adventitia, each layer has been presented and TEM findings were noted in each of the sections regarding CKC, DBC and DBE: intima (Fig. 4), media (Figs. 3, 5), adventitia and adiposa in the descending aorta (Figs. 1-3, 6-10). It is interesting 


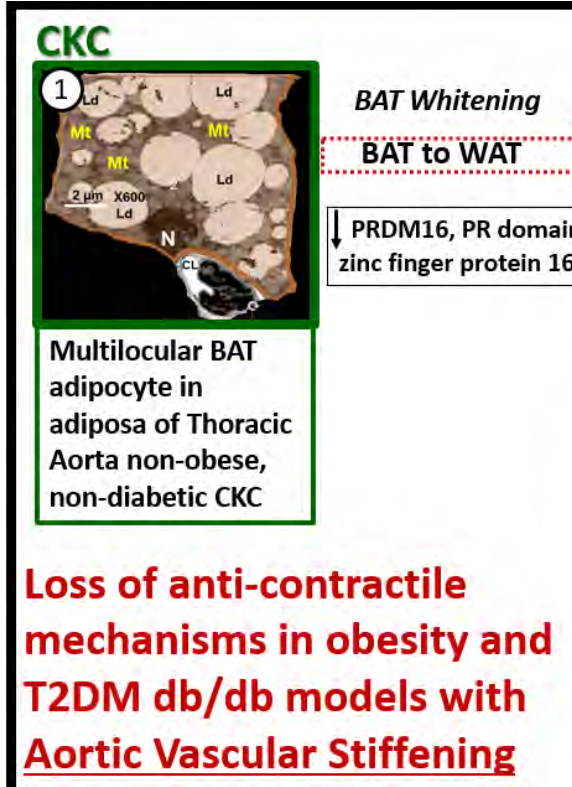

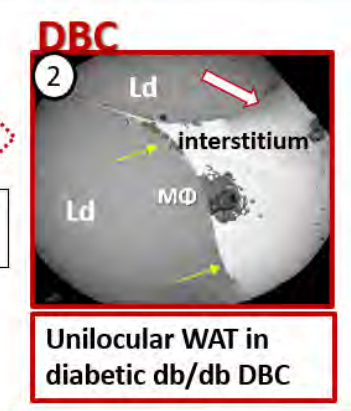

DBC
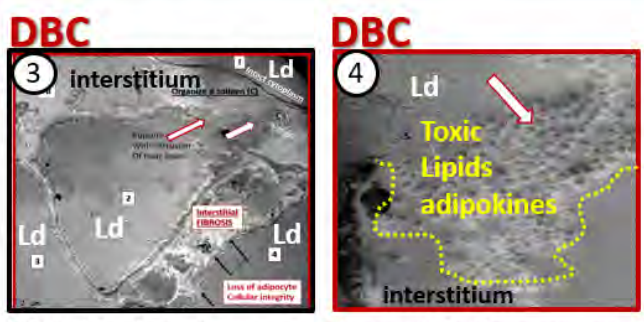

WAT thinning plasma membrane thinning, rupture and extrusion of toxic lipids inciting inflammation.

\begin{tabular}{|l|}
\hline Glucotoxicity \\
Lipotoxicity ROS \\
RAAS Activation with \\
Increase in ANG II and ALDO \\
ALDO \\
Dysfunction of IRS-1 \\
Leptin resistance \\
Insulin resistance \\
HYPOXIA and Chronic Inflammation \\
\hline
\end{tabular}

HYPOXIA and Chronic Inflammation

Hypoxia, oxidative stress and inflammation: Toxic Triad.

Figure 7. A spectrum of phenotypic remodeling from CKC control to obese, $d b / d b$ DBC. This illustration utilizing transmission electron micrographs from the CKC and DBC models assist in illustrating the spectrum of adipose tissue and adipocyte plasticity in these models and provides a possible sequence of events (1 through 4). Some of the major toxicities are listed in the lower right-hand box plus the emerging role of PRDM16 domain. Aldo, aldosterone; Ang II, angiotensin II; BAT, brown adipose tissue; $C K C=$ control heterozygous model; $\mathrm{DBC}=\mathrm{db} / \mathrm{db}$ diabetic models; Ins, insulin; IRS-1, Insulin receptor substrate-1; Ld, lipid droplets; MФ. macrophage; Mt. mitochondria; PRDM16, PR domain zinc finger protein 16; Red open arrows, rupture of plasma membranes; ROS, reactive oxygen species; T2DM, type 2 diabetes mellitus; WAT, white adipose tissue.

that there was no observable increased thickness or compactness in the adventitia tunica compacta similar to the previous Ren2 lean hypertensive model (29); however, the injury mechanisms were different in that this DBC model as the C57BLKS/J background did not demonstrate nearly the elevated blood pressure at this age of 20 weeks when compared to the much younger (9-10-weeks of age) Ren2 lean hypertensive models. Additionally, others have noted that $\mathrm{db} / \mathrm{db}$ models do not always have hypertension and further, hypertension is known to be age-dependent and may also depend on the background upon which the genetic model is created (30).

The DBC did demonstrate obesity and T2DM and all of its associated injury mechanisms similar to the diet-induced obesity Western as well as insulin resistance and of course the marked hyperglycemic - glucotoxic phenotype injury model $(16,31)$. Previously, mean glucose ranges have been shown to be in the range of $100 \mathrm{mg} / \mathrm{dL}$ in the heterozygous lean non-diabetic (CKC) models; $500 \mathrm{mg} / \mathrm{dL}$ for the homozygous obese, insulin resistant and diabetic $d b / d b$ (DBC) male and female models and in the $200 \mathrm{mg} / \mathrm{dL}$ range in the EMPA treated models of the $d b /$ $d b$ mice $(16,31)$.

Empagliflozin treatment protected the BAT from differentiating to WAT and we know at least one of the mechanisms was by lowering the toxicities of hyperglycemia from a mean of $500 \mathrm{mg} / \mathrm{dL}$ range of glucose to a mean of around $200 \mathrm{mg} / \mathrm{dL}$ in the DBE as compared to the mean of $100 \mathrm{mg} / \mathrm{dL}$ in the CKC $(16,31)$. In doing so, EMPA also improved vascular stiffening of the aorta and thus protected the multiple target end-organs known to affect T2DM. This is possible by protecting the adiposa and its BAT protective anti-inflammatory and anti-obesity functions. These possible mechanisms are thought to include protective anticontractile functions of the adiposa as it is now considered to be a virtual endocrine organ with many protective adipokines and adipocyte-derived vasorelaxant factors that are lost with the differentiation to proinflammatory, procontractile and proatherogenic WAT.

The tunica adiposa is an important vascular structural and functional construct of the wall of descending aorta, which may certainly continue to be the frontier of knowledge since it is able to secrete hundred of adipokines with endocrine and paracrine 


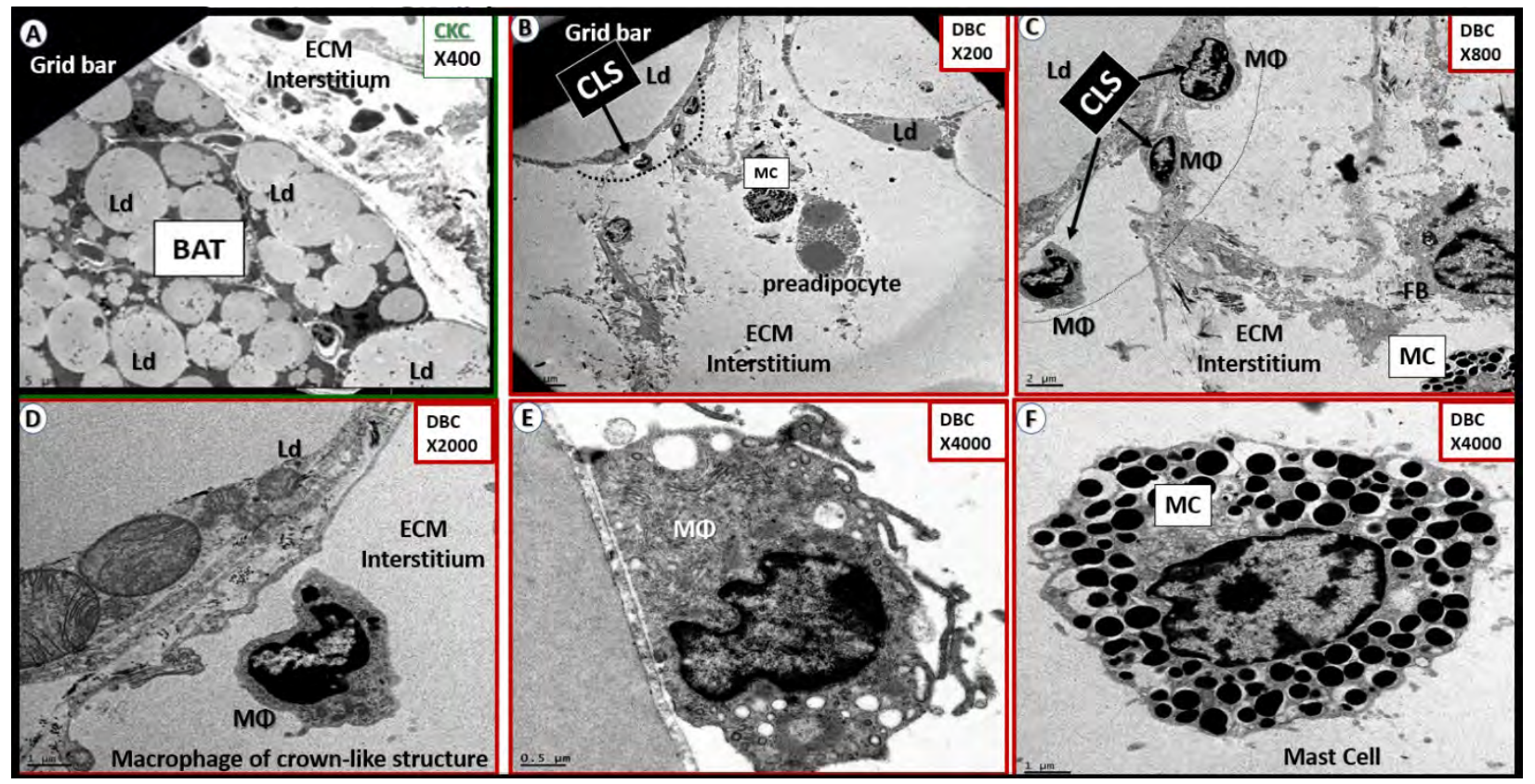

Figure 8. Tunica adiposa is pro-inflammatory in DBC with crown-like structures (CLS) Panel A illustrates the findings of multilocular brown adipose tissue (BAT) without any inflammatory cells. Panels $\mathbf{B}$ and $\mathbf{C}$ depict the accumulation of macrophages $(M \Phi)$ with crown like structures (CLS) in the adiposa. Panels $\mathbf{D}$ and $\mathbf{E}$ each display macrophages and not that in panel $\mathbf{E}$ the actual binding of the macrophage to the plasma membrane of the unilocular adipocyte. Panel $\mathbf{F}$ is a higher magnification of the mast cell (MC) noted in the bottom right of panel $C$. These images depict the multiple labeled inflammatory cells in the adiposa - PVAT of the DBC models with varying magnifications and scale bars labeled. The macrophage with crown-like structures (CLS) was the most common inflammatory cell and included some mast cells, which were only occasionally noted in the control and DBE empagliflozin treated models (DBE). Importantly, there were no CLS in the CKC and DBE. ECM, extracellular matrix; FB, fibroblast; Ld, lipid droplets; Lg, lipid granules; Mt, mitochondria;

systemic and local functions. Adipokines such as adipocytederived vasoactive relaxing factors include the gasotransmitters (nitric oxide and hydrogen sulfide), adiponectin, leptin, hydrogen peroxide, angiotensin 1-7, methyl palmitate and others yet to be possibly identified. Importantly, these anticontractile effects are lost under condition of obesity (32). Also, we have to include the inflammatory, fibrotic and angiogenic adipokines such MCP-1, TNF-alpha, TGF $\beta$, IL-6, IL-1 $\beta$, angiopoietin-like protein 2 and omentin all of which may interact in not only the process of atherogenesis but also in better understand the process of vascular stiffening $(27,31)$.

There are multiple known metabolic toxicities found in the female and male diabetic preclinical DBC and human T2DM subjects, which includes glucotoxicity, advanced glycation end products and its receptor (AGE/RAGE) interaction, lipotoxicity, insulin resistance with hyperinsulinemia, leptin resistance with hyperleptinemia, systemic and local adiposa renin angiotensin aldosterone and receptor (RAAS) activation, hypothalamic pitui- tary adrenal (HPA) dysfunction, oxidative and nitrosative stress (ROS/NOS) activation, chronic inflammatory stress in adiposa and visceral - omental depots, hyperhomocysteinemia and hyperuricemia. These toxicities are capable of acting individually or synergistically and since there are so many variables it is likely that they are constantly working synergistically especially in a genetic leptin receptor deficient model that is constantly stimulated to consume its high caloric intake and driven by hyperleptinemia and leptin resistance with impaired leptin cellular signaling as a result of the point mutation of the leptin receptor in this $d b / d b$ DBC model. In addition to the these metabolic toxicities there may be a central mechanism in regards to tunica adiposa dysfunction that may be referred to as an 'obesity triad', which consists of adiposa hypoxia due to adipocyte expansion and hypertrophy, inflammation as demonstrated in the CLS and oxidative stress, which has been demonstrated in $d b / d b$ models $(2,33)$.

Certain limitations apply to this study including the following this study was limited to immediate immersion fixation as 


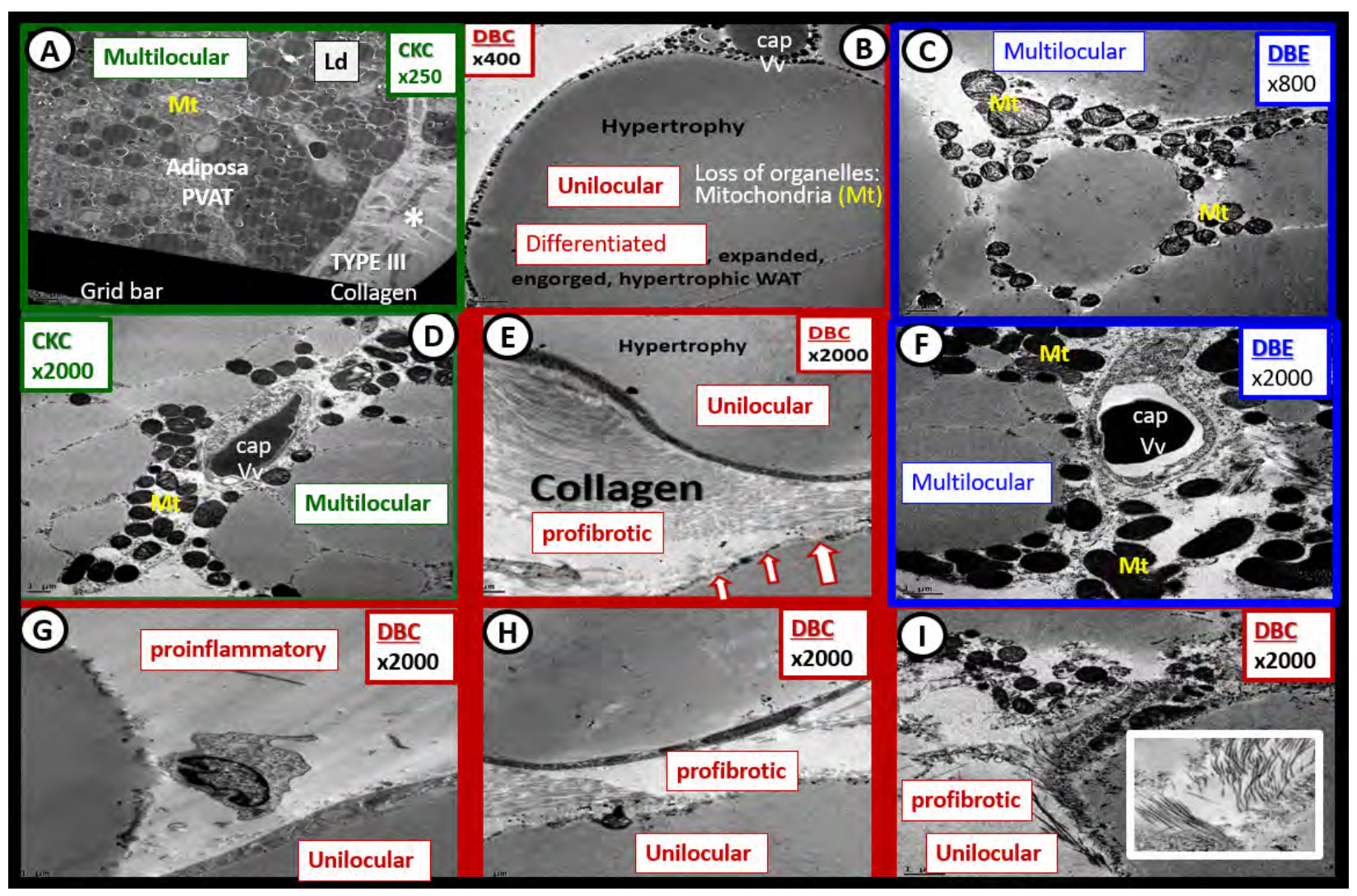

Figure 9. Diabetic $\boldsymbol{d} \boldsymbol{b} / \boldsymbol{d} \boldsymbol{b} \mathrm{DBC}$ are inflammatory and profibrotic. This multi-paneled image depicts the mostly the profibrotic findings in the DBC in red outlines. Importantly, empagliflozin treatment (panels $\mathbf{C}$ and $\mathbf{F}$ outlined in blue) was observed to protect the differentiation of the BAT (multilocular) to WAT unilocular adipocytes as in the DBC (panels B, F, G, and H). Panels E, $\mathbf{G}$ and $\mathbf{H}$ demonstrate the vulnerable (prone to rupture) disrupted adipocyte with loss of cellular integrity with loss of organelles to produce energy and provide proper signaling. Cap = capillary; Ld =lipid $\operatorname{droplet(s);~} V$ = capillary vasa vasorum.

would be done with human autopsy or biopsy specimens; ii. this study was limited to observational findings without quantification; iii. while this study is only a snapshot in time it does allow for the comparison of control CKC to the obese diabetic DBC and to the DBC treated with empagliflozin DBE at the same point in time. Importantly, this study is the same $d b / d b$ model as in the studies of aortic vascular stiffness of the DBC at 20-weeks of age (2) and a four-week younger model at 16-weeks of age (33), which does allow for some functional correlations as well as these ultrastructural observations.

The question often arose during this study of the subcutaneous and visceral adipose depots: Why are subcutaneous adipose depots resistant to inflammation and the visceral adipose tissue including aortic WAT adiposa depot prone to inflammation? The answers must certainly lie in their anatomic location and their primary importance to the tissues they serve in addition to possible differences of genetic - epigenetic programs available in these specific depots (34). Throughout this paper author has used the term differentiation of BAT in control CKC to WAT in the DBC and many refer to this phenomenon as BAT whitening $(35,36)$.

\section{Conclusion}

The diabetic $d b / d b$ DBC has been able to demonstrate both expected and novel findings in the adiposa and these findings will help to better understand the effects of obesity, insulin/leptin resistance and T2DM regarding not only the adiposa but also the descending thoracic aorta and ultrastructural remodeling effects of each tunica of the aorta that accompany aortic vascular stiffening - stiffness with significant kidney and brain remodeling $(2,37)$. It is hopeful that the crossover of knowledge between these ultrastructural images and the discipline of functional molecular biology may lead to advances in the study of cardiometabolic adipobiology (38-40). 


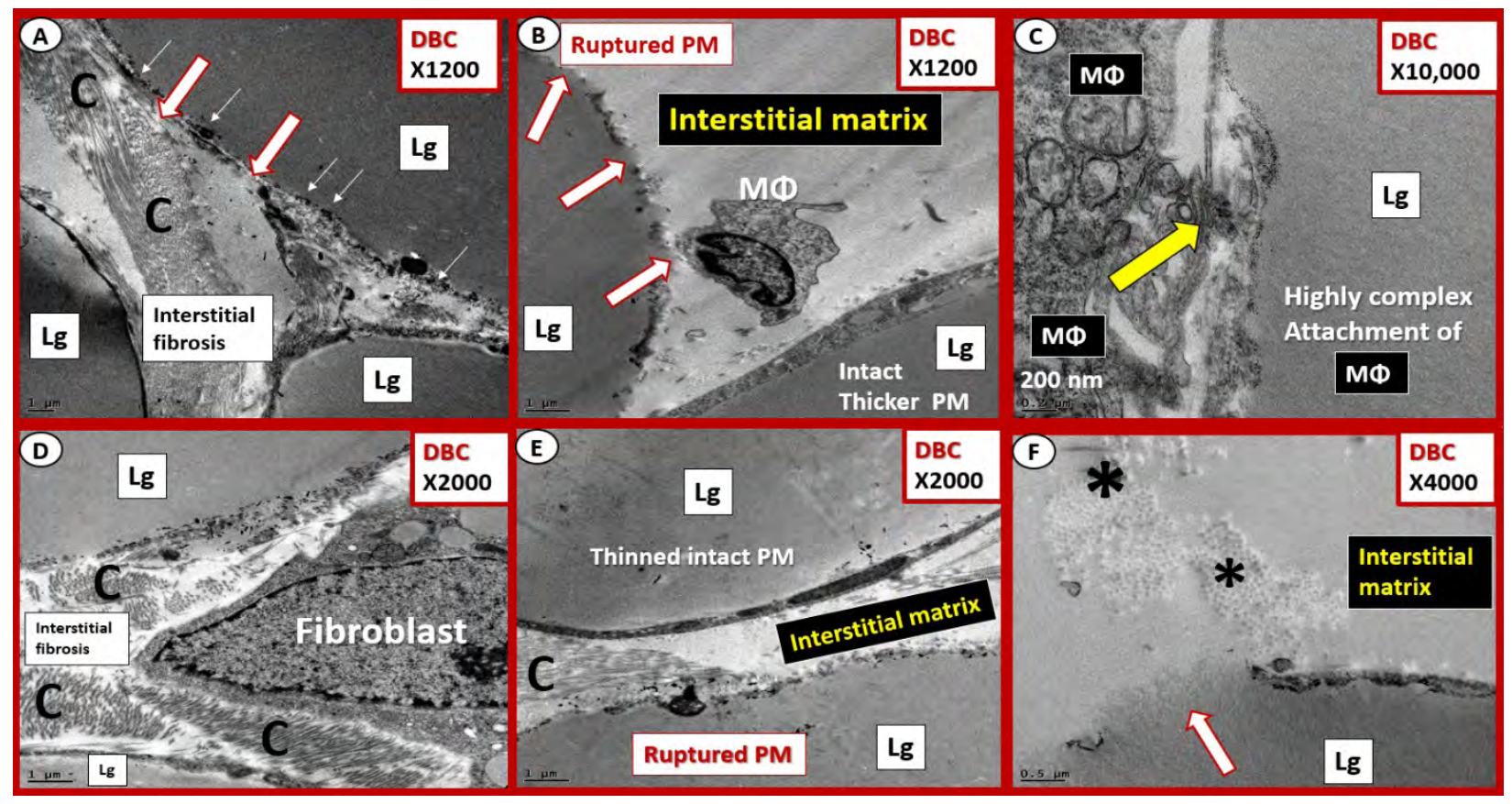

Figure 10. Diabetic DBC adiposa is proinflammatory and profibrotic. All panels actually depict thinned plasma membranes of lipid granules ( $\mathrm{Lg}$ ) in DBC. Panel A illustrates plasma membrane thinning and the upper one depicts plasma membrane (PM) rupture with electron densities suggestive of calcium and possible calcium soaps within the PM (red open arrows). Further, PM rupture is evident (open arrows) and the marked electron densities within the PM (closed arrows may represent calcium soaps (calcium + PM lipids and proteins and thus excessive electron densities which have been shown to represent positive von Kossa staining. Panel B illustrates a macrophage (MФ) representative of inflammation, while panel C depicts the complex attachment of the $M \Phi$ attaching to the PM of the adipocyte. Panel D shows marked fibrosis with collagen $(C)$ within the interstitial matrix in both horizontal and cross section as well as a fibroblast responsible for its synthesis. Panel E also demonstrates organized collagen - fibrosis within the interstitium, while panel $\mathbf{F}$ depicts the very classical image of lipids that have escaped the lipid granule ( $\mathrm{Lg}$ ) due to rupture of its PM (open arrow). Various magnifications in upper right while scale bars appear in lower left of each panel.

\section{Acknowledgement}

The author would like to acknowledge DeAna G. Grant of the Electron Microscopic Core Center at the University of Missouri, Columbia, Missouri for sample preparation and assistance. Female heterozygous littermate controls and (female (C57BLKS/J) and $d b / d b$ (BKS.Cg-Dock7m+/+Leprdb/J) mice were kindly provided by Vincent G DeMarco PhD and Annayya R. Aroor MD, PhD kindly obtained descending thoracic aortas at time of sacrifice.

\section{Competing Interests}

The author declares no competing interests.

\section{References}

1. Ginsberg HN, MacCallum PR. The obesity, metabolic syndrome, and type 2 diabetes mellitus pandemic: Part I. In- creased cardiovascular disease risk and the importance of atherogenic dyslipidemia in persons with the metabolic syndrome and type 2 diabetes mellitus. J Cardiometab Syndr 2009;4(2):113-9. doi: 10.1111/j.1559-4572.2008.00044.x.

2. Aroor AR, Das NA, Carpenter AJ, et al. Glycemic control by the SGLT2 inhibitor empagliflozin decreases aortic stiffness, renal resistivity index and kidney injury. Cardiovasc Diabetol. 2018;17(1):108. doi: 10.1186/s12933-018-0750-8

3. Hayden MR, Tyagi SC. Neural redox stress and remodeling in metabolic syndrome, type 2 diabetes mellitus, and diabetic neuropathy. Med Sci Monit. 2004;10(12):RA291-307. PMID: 15567993

4. Yang Y, Hayden MR, Sowers S, Bagree SV, Sowers JR. Retinal redox stress and remodeling in cardiometabolic syndrome and diabetes. Oxid Med Cell Longev. 2010;3(6):392403. doi:10.4161/oxim.3.6.14786 
5. Jia G, Hill MA, Sowers JR. Diabetic Cardiomyopathy An Update of Mechanisms Contributing to This Clinical Entity. Circ Res 2018;122:624-638. doi: 10.1161/CIRCRESAHA.117.311586

6. Hayden MR, Whaley-Connell A, Sowers JR. Renal Redox Stress and Remodeling in Metabolic Syndrome, Type 2 Diabetes mellitus, and Diabetic Nephropathy: Paying Homage to the Podocyte. Am J Nephrol 2005;25:553-569. https://doi. org/10.1159/000088810

7. Sowers KM Habibi J, Hayden MR. Diabetic Nephropathy and Tubulointerstitial Fibrosis in Cardiometabolic Syndrome and Type 2 Diabetes Mellitus. Journal of the CardioMetabolic Syndrome. 2007; 2(2):143-8. doi: 10.1111/j.15594564.2007.06160.x

8. Hayden MR, Tyagi SC. Is type 2 diabetes mellitus a vascular disease (atheroscleropathy) with hyperglycemia a late manifestation? The role of NOS, NO, and redox stress. Cardiovasc Diabetol. 2003;2:2. doi:10.1186/1475-2840-2-2

9. Hayden MR, Banks WA, Shah GN, Gu Z, Sowers JR. Cardiorenal metabolic syndrome and diabetic cognopathy. Cardiorenal Med 2013;3(4):265-82. doi: 10.1159/000357113

10. Chaldakov GN, Beltowsky J, Ghenev PI, Fiore M, Panayotov P, Rančič G, Aloe L. Adipoparacrinology - vascular periadventitial adipose tissue (tunica adiposa) as an example. Cell Biol Int 2012;36(3):327-30. doi: 10.1042/CBI20110422

11. Chaldakov GN, Fiore M, Ghenev PI, Beltowski J, Ranćić G, Tunçel N, Aloe L. Triactome: neuro-immune-adipose interactions. Implication in vascular biology. Front Immunol 2014;5:130. doi: 10.3389/fimmu.2014.00130

12. Chaldakov GN, Stankulov IS, Fiore M, Ghenev PI, Aloe L, et al. Adipoendocrinology and adipoparacrinology: Emerging fields of study on the adipose tissue. Biomed Rev 2001; 12: 31-39.

13. Jia G, Aroor AR, Sowers JR. Arterial Stiffness: A Nexus between Cardiac and Renal Disease. Cardiorenal Med 2014;4(1):60-71. doi: 10.1159/000360867

14. Markus MRP, Rospleszcz S, et al. Glucose and insulin levels are associated with arterial stiffness and concentric remodeling of the heart. Cardiovasc Diabetol 2019;18(1):145. doi: 10.1186/s12933-019-0948-4

15. DeMarco VG, Habibi J, Jia G, et al. Low-Dose Mineralocorticoid Receptor Blockade Prevents Western Diet-Induced Arterial Stiffening in Female Mice. Hypertension 2015;66(1):99107. doi: 10.1161/HYPERTENSIONAHA.115.05674

16. Hayden MR, Grant DG, Aroor AR, DeMarco VG. Empaglifozin ameliorates type 2 diabetes-induced ultrastructural remodeling of the neurovascular unit and neuroglia in the female db/db mouse. Brain Sci 2019;9(3):E57. doi: 10.3390/ brainsci9030057

17. Romier B, Ivaldi C, Sartelet H, et al. Production of ElastinDerived Peptides Contributes to the Development of Nonalcoholic Steatohepatitis. Diabetes 2018;67(8):1604-1615. doi: $10.2337 / \mathrm{db} 17-0490$

18. Foote CA, Castorena-Gonzalez JA, Ramir-Perez FI, et al. Arterial Stiffening in Western Diet-Fed Mice Is Associated with Increased Vascular Elastin, Transforming Growth Factor- $\beta$, and Plasma Neuraminidase. Front Physiol 2016;7:285. doi: 10.3389/fphys.2016.00285

19. Hayden MR. Ultrastructure study of the transgenic Ren2 rat aorta- Part 2: Media, external elastic lamina and adventitia. Biomed Rev 2019;30:111-123.

20. Chronopoulos A, Tang A, Beglova E, Trackman PC, Roy S. High glucose increases lysyl oxidase expression and activity in retinal endothelial cells: mechanism for compromised extracellular matrix barrier function. Diabetes 2010;59(12):3159-66. doi: 10.2337/db10-036

21. Padilla J, Jenkins NT, Vieira-Potter VJ, Laughlin MH. Divergent phenotype of rat thoracic and abdominal perivascular adipose tissues. Am J Physiol Regul Integr Comp Physiol. 2013;304(7):R543-52. doi: 10.1152/ajpregu.00567.2012

22. Bostick B, Habibi J, Ma L, Aroor A, Rehmer N, Hayden MR, Sowers JR. Dipeptidyl peptidase inhibition prevents diastolic dysfunction and reduces myocardial fibrosis in a mouse model of Western diet induced obesity. Metabolism. 2014;63(8):1000-11. doi: 10.1016/j.metabol.2014.04.002.

23. Grigoraş A, Amalinei C, Balan RA, Giuşcă SE, Avădănei ER, Lozneanu L, Căruntu ID. Adipocytes spectrum - From homeostasia to obesity and its associated pathology. Ann Anat 2018;219:102-120. doi: 10.1016/j.aanat.2018.06.004.

24. Giordano A, Murano I, Mondini E, et al. Obese adipocytes show ultrastructural features of stressed cells and die of pyroptosis. J Lipid Res 2013;54(9):2423-2436. doi: 10.1194/jlr. M038638

25. Akoumianakis I, Tarun A, Antoniades C. Perivascular adipose tissue as a regulator of vascular disease pathogenesis: identifying novel therapeutic targets. British Journal of Pharmacology 2017; 174 3411-3424. doi: 10.1111/bph.13666

26. Henrichot E, Juge-Aubry CE, Pernin A, et al. Production of chemokines by perivascular adipose tissue: a role in the pathogenesis of atherosclerosis? Arterioscler Thromb Vasc Biol 2005; 25: 2594-2599. doi:10.1161/01. ATV.0000188508.40052.35_

27. Aroor AR, Jia G, Sowers JR. Cellular mechanisms underlying obesity-induced arterial stiffness. Am J Physiol Regul Integr Comp Physiol. 2018;314(3):R387-R398. doi: 10.1152/ ajpregu.00235.2016 
28. Nosalski R, Guzik TJ. Perivascular adipose tissue inflammation in vascular disease. Br J Pharmacol 2017;174(20):34963513. doi: 10.1111/bph.13705

29. Hayden MR, Habibi J, Joginpally T, Karuparthi PR, Sowers JR. Ultrastructure Study of Transgenic Ren2 Rat Aorta - Part 1: Endothelium and Intima. Cardiorenal Med 2012;2(1):6682. doi:10.1159/000335565

30. Senador D, Kanakamedala K, Irigoyen MC, Morris M, Elased KM. Cardiovascular and autonomic phenotype of db/db diabetic mice. Exp Physiol 2009;94(6):648-658. doi: 10.1113/expphysiol.2008.046474

31. Lin B, Koibuchi N, Hasegawa Y, Sueta D, Toyama K, Uekawa $\mathrm{K}$, et al. Glycemic control with empagliflozin, a novel selective SGLT2 inhibitor, ameliorates cardiovascular injury and cognitive dysfunction in obese and type 2 diabetic mice. Cardiovasc Diabetol 2014;13:148. doi: 10.1186/s12933-0140148-1

32. Xia N, Li $\mathrm{H}$. The role of perivascular adipose tissue in obesity-induced vascular dysfunction. $\mathrm{Br} J$ Pharmacol. 2017;174(20):3425-3442. doi: 10.1111/bph.13650

33. Habibi J, Aroor AR, Sowers JR, et al. Sodium glucose transporter 2 (SGLT2) inhibition with empagliflozin improves cardiac diastolic function in a female rodent model of diabetes. Cardiovasc Diabetol 2017;16(1):9. doi: 10.1186/ s12933-016-0489-z.
34. Blirando K. Epigenetic regulation of adipocytes phenotype: Implicaton for pervascular adipose tissue contribution to cardiometabolic diseases. Adipobiology 2016,8:19-34.

35. Shimizu I, Aprahamian T, Kikuchi R, et al. Vascular rarefaction mediates whitening of brown fat in obesity. J Clin Invest 2014;124(5):2099-2112. doi: 10.1172/JCI71643.

36. Kotzbeck P, Giordano A, Mondini E, et al. Brown adipose tissue whitening leads to brown adipocyte death and adipose tissue inflammation. J Lipid Res 2018;59(5):784-794. doi: 10.1194/jlr.M079665

37. Hayden MR. Type 2 diabetes mellitus increases the risk of late-onset Alzheimer's disease: Ultrastructural remodeling of the neurovascular unit and diabetic gliopathy. Brain Sci 2019;9(10).pii: E262. doi: 10.3390/brainsci9100262

38. Chaldakov GN. Cardiovascular adipobiology: a novel. Heart-associated adipose tissue in cardiovascular disease. Ser J Exp Clin Res 2008; 9: 81-88.

39. Chaldakov GN, Tonchev AB, Fiore M, Hristova MG, Pancheva R, Rancic G, Aloe L. Implications for the future of obesity management. In: Frühbeck G, editor. Peptides in Energy Balance and Obesity. CAB International 2009; 369-389.

40. Wojcicka G, Jamroz-Wisniewska A, Attanasova P, Chaldakov GN, Chylinska-Kula B, Beltowski J. Differential effects of statins on endogenous $\mathrm{H}_{2} \mathrm{~S}$ formation in perivascular adipose tissue. Pharmacol Res 2011;63:68-76. doi: 10.1016/j. phrs.2010.10.011 